\title{
Reconstruction of temporal activity of microRNAs from gene expression data in breast cancer cell line
}

\author{
Naresh Doni Jayavelu* and Nadav Bar
}

\begin{abstract}
Background: MicroRNAs (miRNAs) are small non-coding RNAs that regulate genes at the post-transcriptional level in spatiotemporal manner. Several miRNAs are identified as prognostic and diagnostic markers in many human cancers. Estimation of the temporal activities of the miRNAs is an important step in the way to understand the complex interactions of these important regulatory elements with transcription factors (TFs) and target genes (TGs). However, current research on miRNA activities excludes network dynamics from the studies, disregarding the important element of time in the regulatory network analysis.

Results: In the current study, we combined experimentally verified miRNA-TG interactions with breast cancer microarray TG expression data to identify key miRNAs and compute their temporal activity using network component analysis (NCA). The computed activities showed that miRNAs were regulated in a time dependent manner. Our results allowed constructing a synergistic network of miRNAs using the computed miRNA activities and their shared regulation of TGs. We further extended this network by incorporating miRNA-TG, miRNA-TF, TFmiRNA and TF-TG regulations in the context of breast cancer. Our integrated network identified several miRNAs known to be involved in breast cancer regulation and revealed several novel miRNAs. Our further analysis detected substantial involvement of the miRNAs miR-324, miR-93, miR-615 and miR-1 in breast cancer, which was not known previously. Next, combining our integrated networks with functional annotation of differentially expressed genes resulted in new sub-networks. These sub-networks allowed us to identify the key miRNAs and their interactions with TFs and TGs of several biological processes involved in breast cancer. The identified markers are validated for their potential as prognostic markers for breast cancer through survival analysis.
\end{abstract}

Conclusions: Our dynamical analysis of the miRNA interactions greatly helps to discover new network based markers, and is highly applicable (but not limited) to cancer research.

Keywords: Network component analysis, microRNAs, Breast cancer, Activity, Data decomposition, Cancer markers, EGFR signaling, Survival analysis, Kaplan-Meier plots

\section{Background}

Cell functions are exerted through gene regulation in response to external cues. MicroRNAs (miRNAs) and transcription factors (TFs) are key regulators in the gene regulation process $[1,2]$. miRNAs are small $(\sim 22$ nucleotides in length) non-coding RNAs that regulate gene expression post transcriptionally in a sequencespecific manner [3]. Many miRNAs are shown to be

\footnotetext{
* Correspondence: nareshd@chemeng.nnu.no; nadi.bar@chemeng.ntnu.no Department of Chemical Engineering, Norwegian University of Science and Technology, Trondheim N7491, Norway
}

(C) 2016 Jayavelu and Bar. Open Access This article is distributed under the terms of the Creative Commons Attribution 4.0 International License (http://creativecommons.org/licenses/by/4.0/), which permits unrestricted use, distribution, and reproduction in any medium, provided you give appropriate credit to the original author(s) and the source, provide a link to the Creative Commons license, and indicate if changes were made. The Creative Commons Public Domain Dedication waiver (http://creativecommons.org/publicdomain/zero/1.0/) applies to the data made available in this article, unless otherwise stated.

involved in cancer related biological processes, such as cell division, growth, development, apoptosis, proliferation and differentiation [4-7]. Therefore, constructing the miRNAs mediated gene regulation networks by utilizing gene expression data has become a regular practice in today's miRNA research. However, all these studies of miRNA regulatory networks focused on static reconstruction of the miRNA regulatory activities. By doing so, they excluded the important element of time from the network analysis. However, since we know regulatory networks are dynamic (time dependent) by 
nature, important network information in those studies may have been lost.

Several studies applied statistical methods to investigate the role of miRNAs in gene regulatory networks. Madden et al identified key miRNAs associated with diseases through time-independent multivariate statistical analysis [8]. _ENREF_47Liang et al developed a web based tool to compute the microRNA activity from its TG expression data based on the negative regulatory relationship between miRNAs and TGs [9]. Mezlini et al developed a regression model to identify key miRNAs and their activity from TG expression and miRNA-TG network [10]._ENREF_47_ENREF_44 The approach proposed by Cheng et al [11] computed a series of static miRNA activities using the differential expression values of the TGs at each time point. Although their approach appears to construct a timeseries miRNA activity profiles, it considers each time point regardless of the expression levels in the other time points. Recently, Schulz et al extended the DREM (Dynamic Regulatory Events Miner) model to mirDREM to reconstruct the dynamic miRNA regulated interaction networks [12]. This model presents the list of significantly pivotal miRNAs and TFs at each time point. However, none of these methods computed the changes in miRNA activity with time.

Network component analysis (NCA) $[13,14]$ is a data decomposition approach that has been successfully employed in several species and in numerous research studies to compute the temporal activity profiles of TFs and construction of dynamic networks [14-28]. The method integrates temporal TG expression data and known network topology. _ENREF_24 In the current study, we exploited this approach for computing the temporal activities of the key miRNAs using only TG expression data (no miRNA data) and experimentally verified miRNA-TG relations. Using the NCA, we identified the key miRNAs, TFs and their activities in epidermal growth factor receptor (EGFR) signaling in breast cancer cells._ENREF_22_ENREF_23 We used the computed miRNAs temporal activities to identify co-regulating miRNAs (synergistic network) that show similar activity patterns and co-regulating common TGs, and validated these miRNAs with a literature study. Additionally, we built an integrated network of miRNAs, TFs and their TGs by retrieving miRNA-miRNA, miRNA-TG, TF-TG and TF-miRNA interactions from literature and combining these with the results of the NCA. With this approach, we identified several miRNAs that were known to be involved in regulation in breast cancer cells, and we revealed several novel miRNAs that are most likely to be involved in breast cancer, but were not known previously. These miRNAs can potentially serve as breast cancer markers.

\section{Results}

Our approach for reconstructing the miRNA temporal activity from its TG expression using NCA is presented in Fig. 1a and complete details are described in the Methods section.

\section{Dynamics of miRNA activity}

The activity profiles (normalized) of several miRNAs which are already known to be involved in breast cancer cells are presented in Fig. 1b. Although the miRNAs showed activity at all-time points, peak activity is demonstrated at 1 or 2 time points. The miRNA let-7a-5p displayed increasing repressing activity with time. This miRNA is known to be a tumor suppressor regulating many genes that inhibit cell migration in breast cancer [29]. The miRNA miR-18b-5p is also involved in breast cancer, regulating genes involved in cell migration and metastasis [30]. This miRNA showed peak activation at $8 \mathrm{~h}$ in the current study. The miRNA let-7d-5p showed a peak repressing activity around $2 \mathrm{~h}$ after EGF treatment and it is aberrantly expressed in breast cancer cells in previous study [31]. The miRNA miR-20a-5p displayed a peak repressing activity at $10 \mathrm{~min}$ and this miRNA also involved in previous breast cancer studies [32]. Yu et al showed that miR-20a-5p and miR-17-5p suppressed the breast cancer cell proliferation by negatively regulating the gene cyclin D1 [32]. The miRNA miR-30a-5p is identified to be a novel prognostic marker in breast cancer in several past studies [33-35] and it showed a peak repression very late (around $36 \mathrm{~h}$ ) in the current study. The miRNA miR-200c-3p also displayed a very late activation at $36 \mathrm{~h}$ and it is involved in regulating epithelial to mesenchymal transition (EMT) by targeting the genes ZEB1 and SIP1 in breast cancer in response to transforming growth factor (TGF) [36]. The miRNA miR-155-5p demonstrated periodic peak activations at $10 \mathrm{~min}, 2$ and $24 \mathrm{~h}$. This miRNA is also known to be involved in the previous breast cancer studies with roles in cell survival, growth and chemosensitivity $[37,38]$. The miRNA miR-210-3p exhibited peak repression activities very early at $15 \mathrm{~min}$ and late during 8-36 h time period and this miRNA has been identified as prognostic marker in breast cancer [39]. Next, we used hierarchical clustering to explore groups of miRNAs with similar activity profiles (Fig. 1c). We found two distinctive groups of miRNAs that activates or repress at all time, and two smaller groups of miRNAs that alternate between activation and repression.

The computed activities demonstrated that EGF activated microRNAs in time dependent manner. To further understand the timely cascade of regulation of EGF in breast cancer, we studied the time points of peak activation of the TGs, (from differential expression data) the TFs and the miRNAs, the last two were computed from 
A
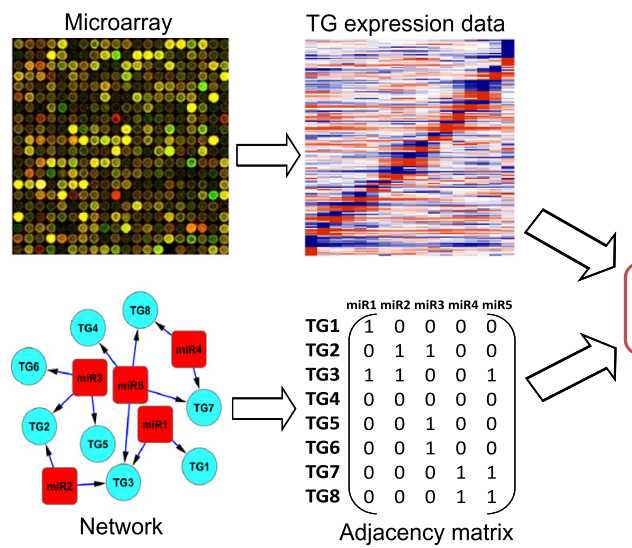

B

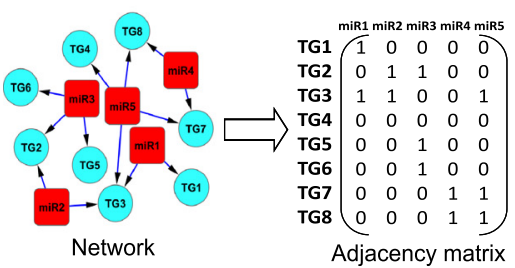

NCA
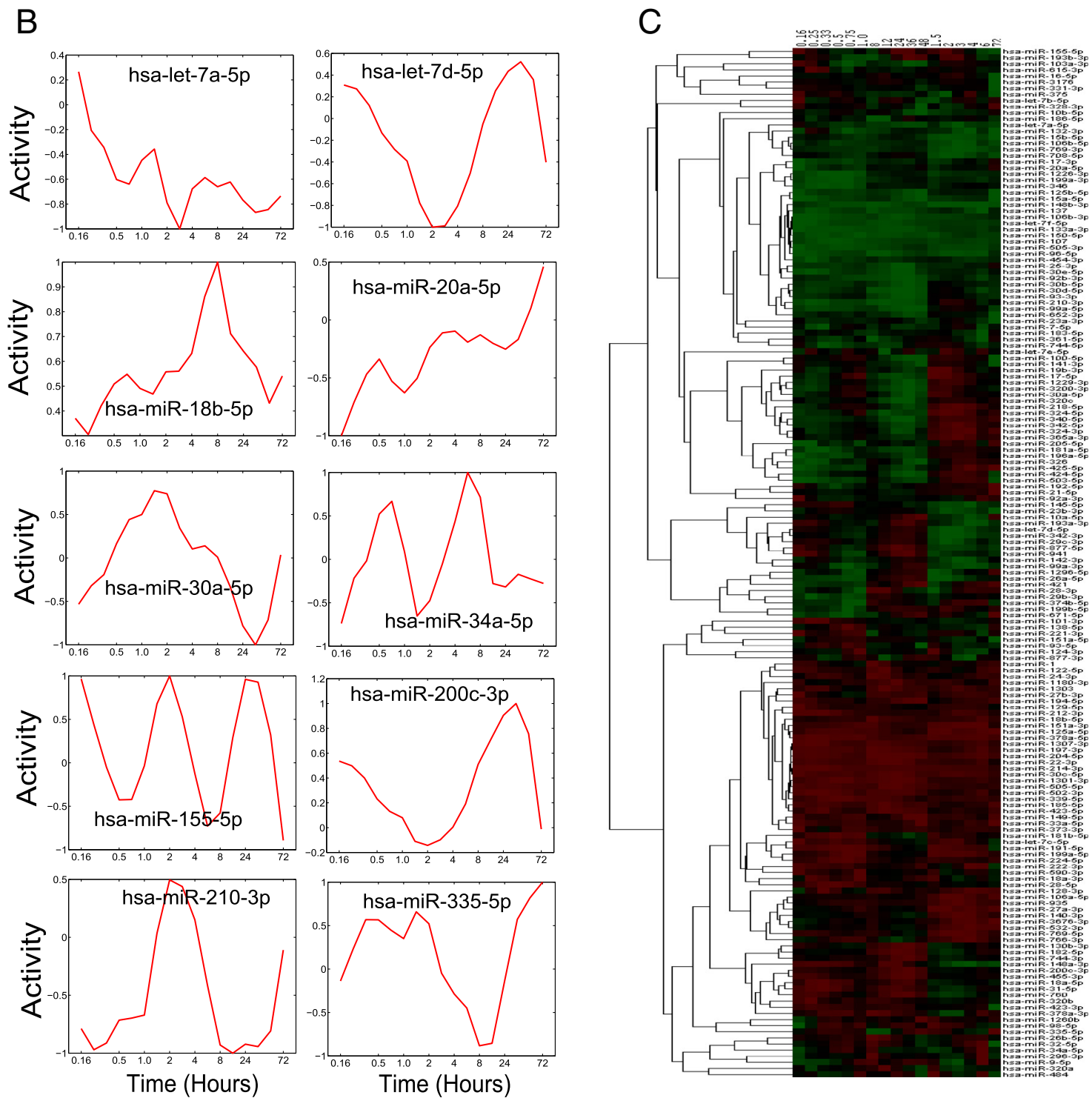

Fig. 1 Schematic of the approach and computed miRNA activities: (a) NCA approach for reconstructing the temporal activities of miRNAs. b Reconstructed temporal activity profiles (normalized values) of selected breast cancer associated miRNAs. c Hierarchical clustering of miRNAs activities. Here, each row represents a target, and the column represents time point 
NCA (Fig. 2). This analysis revealed that EGFR signaling is a highly dynamic process, and the regulation operates in cascades, activating groups of TGs, miRNAs and TFs in a timely manner. The larger effects of EGF stimulation on TGs are observed around $10 \mathrm{~min}, 2 \mathrm{~h} 36 \mathrm{~h}$ and $72 \mathrm{~h}$. Interestingly, for the regulators (miRNAs and TFs) also larger effects are observed at the same time points respectively.

\section{miRNA-miRNA synergistic network}

Synergistic interactions between miRNAs are a key to understand the complex mechanisms of cancers, several miRNAs are usually found together in a particular cancer or disease. In the present study, a miRNA-miRNA synergistic network (MMSN) was constructed based on the computed temporal activities and shared co-regulating TGs (Fig. 3a). In this constructed network, each node represents the miRNA and edges represent similar activity profile (Pearson correlations $>0.7$ ) and at least 3 shared TGs. Thus, the constructed network included 112
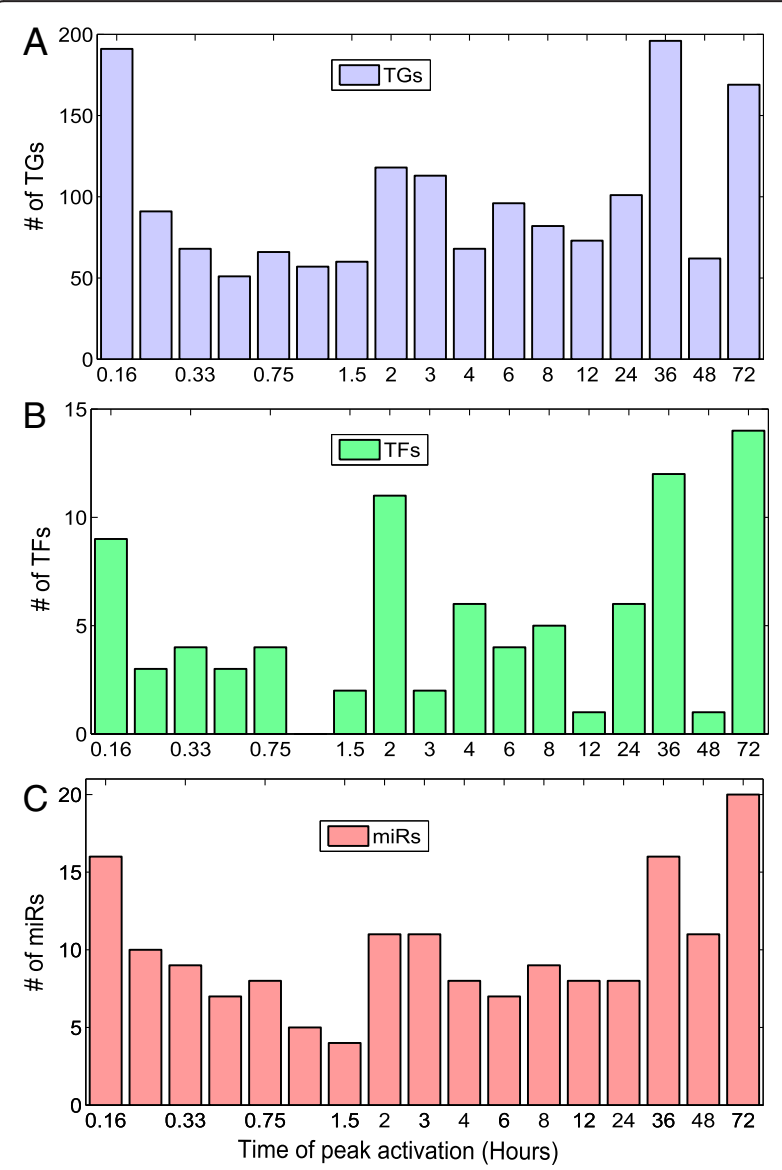

Fig. 2 EGF dynamic regulation: The number of active TGs, TFs and miRNAs at each time point is presented. (a) TGs (b) TFs (c) miRNAs. The active TGs, TFs and miRNAs are defined at each time point based on peak expression or activity at that time point
miRNAs and 314 interactions between the miRNAs (Fig. 3b, Additional file 1). The network followed a power law degree distribution which is a typical biological network property (Fig. 3c). The network is highly interconnected with a clustering coefficient of 0.356 and a mean connectivity of $5.6 .25 \%$ of miRNAs have at least 10 synergistic interactions with other miRNAs. $38 \%$ of the miRNAs in this network were already experimentally verified to be involved in breast cancer studies (according to miRcancer, miR2Disease databases and manual curation $[40,41])$. The miRNAs miR-324-3p, miR-17-5p, miR-30a-5p, miR-93-3p and miR-196a-5p were densely connected, each having more than 17 synergistic interactions with other miRNAs in the network. Furthermore, these miRNAs are interacting with at least 5 known breast cancer associated miRNAs in the network. Of these, miR-17-5p, miR-30a-5p and miR-196a-5p were already known to be involved in breast cancer. To further strengthen our prediction of miRNA-miRNA interactions, we compared the predictions from this study with previously reported data. For this purpose, we downloaded complete miRNA-miRNA regulation data of Sengupta et al [42] and found that more than $80 \%$ (258 out of 314) overlapping interactions between these two studies (Additional file 2). We stress that although we found a large overlap between the two studies, the interactions from Sengupta et al [42] are purely computational, just as our's do, but are independent of ours, taken with a completely different approach. In addition to this, we evaluated the synergy of miRNAs in the current network with randomly generated 100 miRNA-miRNA networks keeping the same node degrees as the original network. The mean value $(0.114)$ for clustering coefficient of the random networks is significantly $(P$-value $<$ $1 \mathrm{e}-10)$ lower than the value $(0.356)$ of the original network.

\section{Analysis of the integrated network in breast cancer}

One of the main objectives of this study is to determine the core regulators of EGFR signaling in breast cancer cells and understand their role in breast cancer. To achieve this goal, we constructed an integrated network of miRNAs, TFs and TGs (Methods, Additional files 1, 2, 3, 4, 5, 6 and 7). The final integrated network followed a power law degree distribution (The network is provided as a cytoscape file (.cys) in Additional file 3). The network includes 168 miRNAs, 328 TFs and 1072 TGs and 20534 interactions. In this network, we find that $87 \%$ of the TFs have at least 3 or more connections. Similarly, $89 \%(150 / 168)$ of the miRNAs regulate at least five TGs and $82 \%$ of TGs are targeted by five or more regulators including both miRNA and TFs. Thus, the integrated regulatory network is complex in terms of 

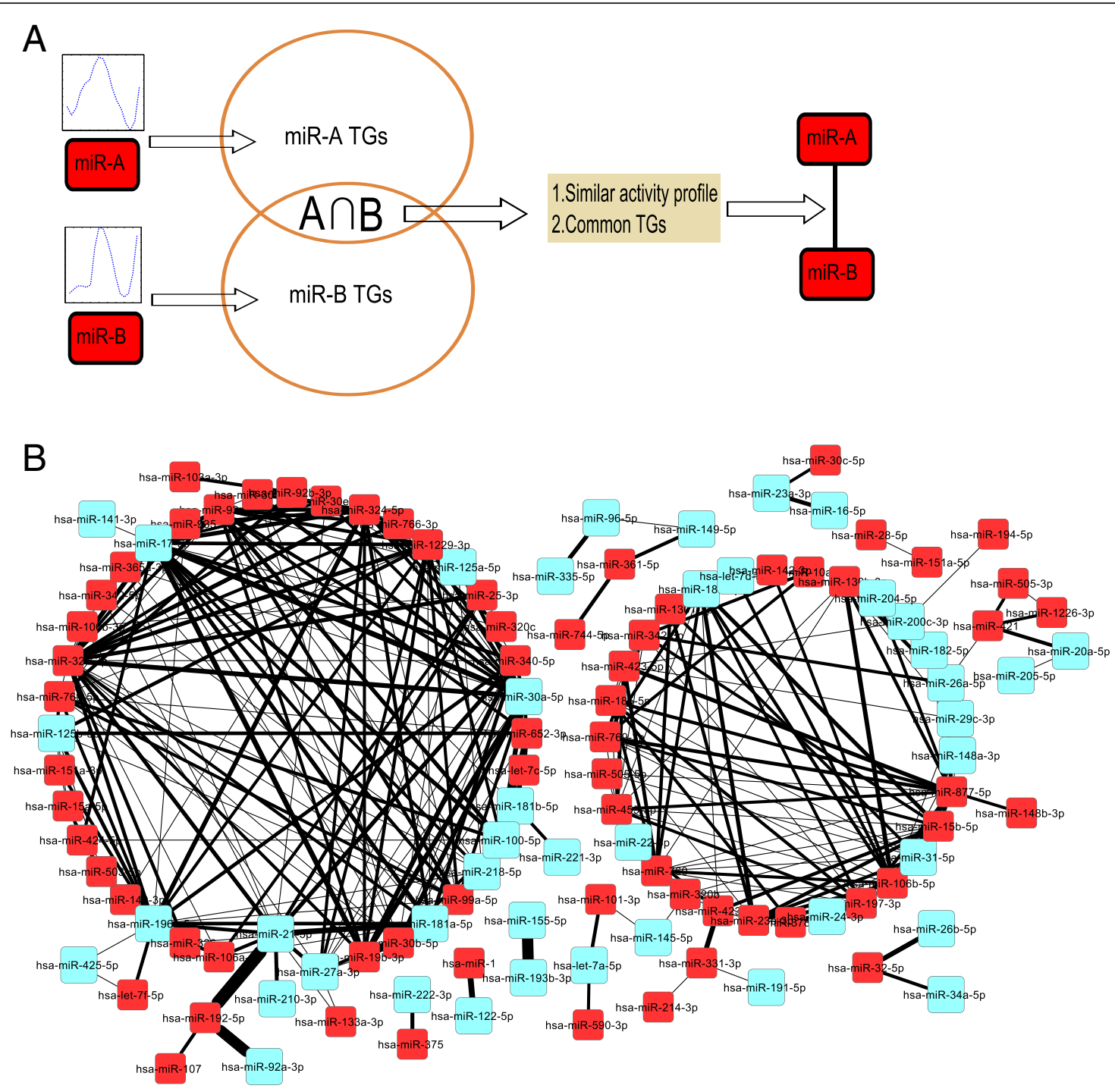

C

Degree distribution
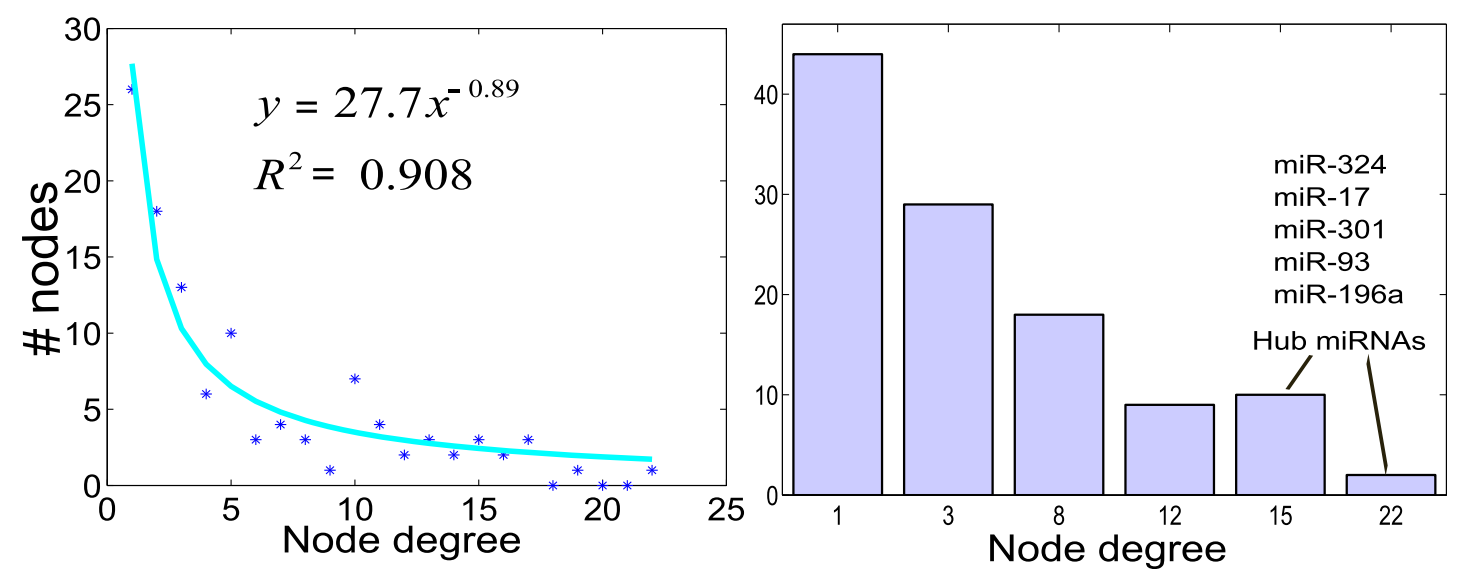

Fig. 3 (See legend on next page.) 
(See figure on previous page.)

Fig. 3 Synergistic network of miRNAs: (a) Schematic of miRNA-miRNA synergistic network construction. b Predicted synergistic interaction pairs of miRNAs in the form of network in breast cancer. Here cyan squares represent breast cancer associated miRNAs in previous studies and red squares represent new miRNAs with no previous involvement in breast cancer studies. c Degree distribution. Right panel: scatter plot of the node degree (number of connections to a specific miRNA) vs. the number of nodes (miRNAs) revealed the power law degree distribution. Left panel: Distribution of miRNA as a function of their node number. We found several hub miRNAs with large number of connections

targets multiplicity and miRNAs co-operativity in the regulation of TGs. The in-degree and out-degree distributions of the network follow a power-law degree distribution with slopes of -1.9 and -4.66 respectively. This indicates that the network is not random but a complex biological network with organized structure [43]. We hypotheses that regulating elements with large number of connections (node degree, whether they are miRNAs or TFs), can be considered as 'core' regulators [43]. In order to identify these core regulators, we focused on hub nodes with the largest number of interactions in the network. The identified top 20 hub miRNAs and TFs are presented in Table 1 and Table 2 respectively. Of these, 14 miRNAs were already known to be involved in breast cancer cells and six were unexplored yet. There were $11 \mathrm{TFs}$ known to be involved in breast cancer, and 9 that were yet to be studied (Table 2).

\section{Functional annotation and pathway analyses of differentially expressed TGs}

To increase our understanding on the role of EGF in breast cancer, we performed functional annotation of the TGs and TFs in the integrated network using functional annotation tool DAVID (Methods). We found that pathways in cancer, TGF-beta signaling pathway, the MAPK signaling pathway, the Wnt signaling pathway, the cell cycle, Notch signaling pathway, melanogenesis, the ErbB signaling pathway and several cancers were all statistically significant affected by the differentially expressed genes in breast cancer cells (Table 3).

Table 1 The top 20 miRNAs with highest degree in breast cancer integrated regulatory network. The degree of a node is the sum of in-coming and out-going connections with other nodes in the network. PMIDs denote the 'pubmed' identification numbers

\begin{tabular}{|c|c|c|c|c|}
\hline Rank & miR Name & Degree & Breast cancer related & PMIDs \\
\hline 1 & hsa-miR-335-5p & 220 & Yes & 18185580 \\
\hline 2 & hsa-miR-124-3p & 185 & Yes & 22333974,22085528 \\
\hline 3 & hsa-miR-26b-5p & 174 & Yes & 23374284,21510944 \\
\hline 4 & hsa-miR-16-5p & 137 & Yes & 22583478 \\
\hline 5 & hsa-let-7b-5p & 114 & Yes & 23339187 \\
\hline 6 & hsa-miR-615-3p & 110 & No & - \\
\hline 7 & hsa-miR-155-5p & 107 & Yes & 23372341,16103053 \\
\hline 8 & hsa-miR-92a-3p & 105 & Yes & 23052693 \\
\hline 9 & hsa-miR-1 & 96 & No & - \\
\hline 10 & hsa-miR-21-5p & 90 & Yes & 23052693, 19419954 \\
\hline 11 & hsa-miR-484 & 88 & No & - \\
\hline 12 & hsa-miR-30a-5p & 86 & Yes & 22476851,23389917 \\
\hline 13 & hsa-miR-193b-3p & 82 & Yes & 19701247 \\
\hline 14 & hsa-miR-34a-5p & 79 & Yes & 23032974 \\
\hline 15 & hsa-miR-17-5p & 78 & Yes & 16940181,20505989 \\
\hline 16 & hsa-miR-192-5p & 77 & No & - \\
\hline 17 & hsa-miR-324-5p & 73 & No & - \\
\hline 18 & hsa-miR-98-5p & 72 & Yes & 18812439 \\
\hline 19 & hsa-miR-324-3p & 71 & No & - \\
\hline 20 & hsa-miR-125b-5p & 64 & Yes & 22693547,21444677 \\
\hline
\end{tabular}


Table 2 The top 20 TFs or TGs with highest degree in breast cancer integrated regulatory network. The degree of a node is the sum of in-coming and out-going connections with other nodes in the network. PMIDs denote the 'pubmed' identification numbers

\begin{tabular}{|c|c|c|c|c|}
\hline Rank & TF Name & Degree & Breast cancer related & PMIDs \\
\hline 1 & SP1 & 371 & Yes & 19812674 \\
\hline 2 & TCF12 & 343 & Yes & 20525248 \\
\hline 3 & SP4 & 337 & No & - \\
\hline 4 & SP3 & 309 & No & - \\
\hline 5 & SP2 & 259 & Yes & Breast cancer database (www.breastcancerdatabase.org) \\
\hline 6 & MYOD1 & 222 & Yes & 20525248 \\
\hline 7 & JUN & 215 & Yes & 20525248 \\
\hline 8 & ERG & 211 & No & - \\
\hline 9 & MYF6 & 199 & No & - \\
\hline 10 & ETV7 & 189 & Yes & 20525248 \\
\hline 11 & ARID5B & 187 & No & - \\
\hline 12 & MYF5 & 183 & No & - \\
\hline 13 & EGR2 & 182 & Yes & 20525248 \\
\hline 14 & MYOG & 182 & No & - \\
\hline 15 & ETS1 & 181 & Yes & 20668451,20525248 \\
\hline 16 & ASCL1 & 180 & No & - \\
\hline 17 & MYC & 178 & Yes & 20525248 \\
\hline 18 & ETS2 & 176 & Yes & Breast cancer database (www.breastcancerdatabase.org) \\
\hline 19 & TCF3 & 174 & No & - \\
\hline 20 & ELF2 & 173 & No & - \\
\hline
\end{tabular}

The significantly enriched biological terms that were identified from the PANTHER database include PDGF signaling pathway, PI3 kinase pathway, JAK/STAT signaling pathway, apoptosis pathway and several overlapping pathways that are identified from KEGG database (Full list is provided in Additional files 4 and 5). The involvement of these pathways in human breast cancers has been described in previous studies [44-47]. This analysis facilitated the identification of previously known and newly

Table 3 Statistically significant biological pathways affected by differentially expressed TGs in breast cancer cells identified from the KEGG database using DAVID

\begin{tabular}{llll}
\hline KEGG ID & Description & \# Genes & P-Value \\
\hline hsa05200 & Pathways in cancer & 69 & $5.51 \mathrm{E}-08$ \\
hsa04350 & TGF-beta signaling pathway & 24 & $3.61 \mathrm{E}-05$ \\
hsa04010 & MAPK signaling pathway & 51 & $6.72 \mathrm{E}-05$ \\
hsa04310 & Wht signaling pathway & 31 & $7.22 \mathrm{E}-04$ \\
hsa04110 & Cell cycle & 25 & 0.003827 \\
hsa04330 & Notch signaling pathway & 11 & 0.027579 \\
hsa04662 & B cell receptor signaling pathway & 15 & 0.030166 \\
hsa04910 & Insulin signaling pathway & 23 & 0.034331 \\
hsa04660 & T cell receptor signaling pathway & 19 & 0.043201 \\
hsa04012 & ErbB signaling pathway & 16 & 0.047711 \\
\hline
\end{tabular}

discovered pathways in breast cancer. Positive and negative regulation of transcription and gene expression, regulation of transcription factor activity, regulation of cell differentiation, proliferation, migration, apoptosis, morphogenesis, angiogenesis and regulation of signal transduction are enriched biological processes (Table 4).

Next, we combined the pathways and gene ontology (GO) results with the integrated network and extracted significantly enriched biological process specific subnetworks (Fig. 4). For instance, Cell-cycle sub-network is mainly regulated by E2F family of TFs (E2F1, E2F2, E2F4, and E2F5), SMAD family of TFs (SMAD2, SMAD3, SMAD4) and miRNAs has-miR-335-5p, hasmiR-26b-5p and has-miR16-5p. Many of these regulators are involved in cell-cycle control. In the similar manner, we constructed the angiogenesis and cell migration subnetworks.

\section{Discussion}

Understanding regulation and precise control of gene expression in higher organisms is a complex process, and miRNAs and TFs are two key regulators of this process. In the current study, we used the well-studied NCA approach to compute the temporal activities of the TFs, and for the first time, for the miRNAs as well. Although several previous studies demonstrated the 
Table 4 Statistically significant biological processes affected by differentially expressed TGs in breast cancer cells identified using DAVID

\begin{tabular}{llll}
\hline GO ID & Description & \# Genes & P-Value \\
\hline GO:0010628 & positive regulation of gene expression & 220 & $2.59 \mathrm{E}-79$ \\
GO:0045941 & positive regulation of transcription & 229 & $4.94 \mathrm{E}-78$ \\
GO:0051173 & positive regulation of nitrogen compound metabolic process & 122 & $1.65 \mathrm{E}-76$ \\
GO:0016481 & negative regulation of transcription & 129 & $3.60 \mathrm{E}-26$ \\
GO:0010629 & negative regulation of gene expression & 66 & $5.17 \mathrm{E}-26$ \\
GO:0045596 & negative regulation of cell differentiation & 64 & $1.18 \mathrm{E}-17$ \\
GO:0045597 & positive regulation of cell differentiation & 139 & $5.79 \mathrm{E}-15$ \\
GO:0042981 & regulation of apoptosis & 72 & $4.34 \mathrm{E}-12$ \\
GO:0008285 & negative regulation of cell proliferation & 73 & $3.28 \mathrm{E}-09$ \\
GO:0008284 & positive regulation of cell proliferation & 28 & $4.58 \mathrm{E}-07$ \\
GO:0051090 & regulation of transcription factor activity & 16 & $9.89 \mathrm{E}-07$ \\
GO:0045787 & positive regulation of cell cycle & 115 & $2.38 \mathrm{E}-04$ \\
GO:0009966 & regulation of signal transduction & 37 & $4.86 \mathrm{E}-04$ \\
GO:0010608 & posttranscriptional regulation of gene expression & $5.93 \mathrm{E}-04$ \\
GO:0000902 & cell morphogenesis & 55 & 31 \\
GO:0030334 & regulation of cell migration & 28 & $8.06 \mathrm{E}-04$ \\
GO:0001525 & angiogenesis & & $8.51 \mathrm{E}-04$ \\
\hline
\end{tabular}

construction of miRNA mediated gene networks, their approaches required the expression data of both miRNAs and TGs. In contrast, our reconstruction approach needs only expression data of TGs. With the publicly available large volumes of the microarray and RNAsequencing (RNA-seq) TG expression data and experimentally verified miRNA-TG data, the NCA approach may serve as a powerful tool to study and understand the miRNA mediated gene regulation. With the computed temporal activities and gene expression data, we are able to identify the time specific active miRNAs, TFs and TGs. This analysis resulted in the identification of EGF stimulation's dominant response at selective time points. Another interesting observation from this analysis is that the number of activated TGs are strongly correlated with their active regulators, TFs (Pearson correlation $=0.815$ ) and miRNAs (Pearson correlation $=$ 0.867 ) over entire time period.

We constructed the miRNA-miRNA synergistic network based on similar temporal activity of miRNAs and their shared TGs. There are several past studies constructed miRNA-miRNA networks but they mostly are based on combinations of shared TGs of miRNA pair, enriched in same gene ontology term, sequence, secondary structure and shared pathways [48-51]. However, none of these studies were used the temporal information knowing that miRNA-TG regulation is highly dynamic. Therefore, the synergistic network constructed in this study is one of the first attempts to incorporate temporal information. This network not only captured synergistic interactions between miRNAs but also identified novel miRNA regulators in breast cancer.

To understand the miRNA regulation more comprehensively, we further extended this synergistic network with TGs and TFs. Further examination of network identified hub miRNAs (hsa-miR-335-5p, hsa-miR-124-3p, hsa-miR-26b-5p, and hsa-miR-16-5p) and TFs (SP1, TCF12, JUN, MYOD1). Most of these hubs are either well-known regulators or are reported to play key roles in breast cancer. For instance, the miRNA miR-335-5p, the top hub node in the network is already known to be a key regulator in suppressing breast cancer metastasis and migration through regulation of targets SOX4 and TNC [52]. The miR-124-3p was shown to be a novel tumor suppressor and a co-regulating EGFR driven cell cycle protein, inhibiting proliferation in breast cancer [53]. The miRNA miR-26b-5p was also shown to be a potential therapeutic target for breast cancer. This miRNA inhibits the cell proliferation by regulating the target PTGS2 [54]. The synthetic growth hormone progestin down regulated the miR-16-5p and cyclin $\mathrm{E}$ was identified as one of its targets in breast cancer [55]. Furthermore, this miRNA inhibited the growth of progestin treated breast cancer cells and thus its role as tumor suppressor. The miRNA let-7b-5p was also shown to have a tumor suppressor role in breast cancer patients with lymph node metastasis, by repressing the expressions of the genes PAK1, DIAPH2, RDX and ITGB8 

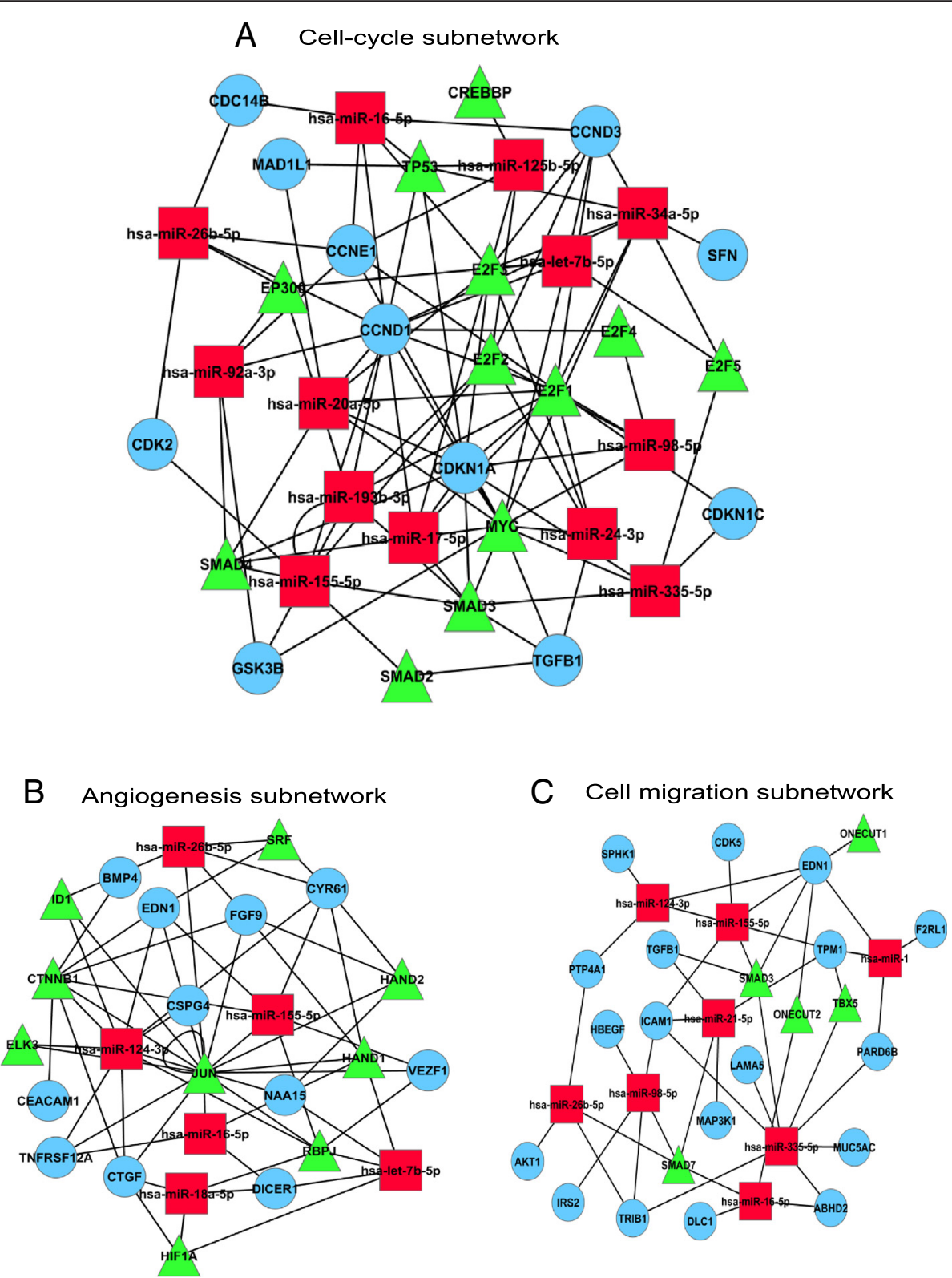

Fig. 4 Sub-networks of biological processes: (a) Cell-cycle sub-network. b Angiogenesis sub-network. c Cell migration sub-network

[29]. The miRNA miR-193b-3p was shown to be an important marker in clinical metastasis of human breast cancer cells, which potentially up-regulates the expression of uPA [56]. In addition to those hub miRNAs, we found hub miRNAs with no previous association in breast cancer, including miR-615-3p, miR-1, miR-484, miR-192-5p and miR-324-5p. We suggest that the novel miRNAs found from our integrated network have potential therapeutic outcomes in breast cancer and should be further explored. Similarly, the top hub TFs we found in the integrated network such as SP1, SP2, TCF12, MYC, JUN and EGR2, were also well-known regulators in breast cancer. Yang et al showed that SP1 and HSF1 play an important role in the regulation of FUT4 (Fucosyltransferase IV), which is associated with breast cancer epithelial cell proliferation [57]. Zhang et al identified that oncoprotein HBXIP activates the gene PDGFB through transcription factor SP1, to promote proliferation in breast cancer cells [58]. Chen et al showed that JUN miR-21 activates Bcl-2 expression and thus promotes chemo resistance in triple negative breast cancer cells [59]. The TF ETS1 promotes proliferation, migration and invasion through stimulation of estrogen receptor alpha $(E R \alpha)$. Verschoor et al showed the ETS1 involvement in energy metabolism and oxidative stress in breast and ovarian cancers [60]. 
To further validate our identified markers both miRNAs and TFs, we performed survival analysis with the publicly available clinical data to uncover their role in breast cancer survival outcome (see Methods for details). Figure 5 presents the Kaplan-Meier plots for the miRNAs and TFs, selected from Tables 1 and 2, respectively (see Additional file 5 for the remaining miRNAs and TFs). The patients with high expression of miR-335 and miR-16 had significantly better survival rates compared to patients with low expression. Similar findings are observed with the TFs SP1 and MYOD1. We suggest that these miRNAs and TFs can potentially serve as positive prognostic markers in breast cancer. We note that although the majority of the markers presented in this study (Tables 1 and 2) were identified as markers with potentially better survival rates, few markers did not demonstrated in the survival analysis any better performance. For instance, TCF12 which was identified as a marker in this study did not show better survival (see Additional file 6A).

We further analyzed the TGs and TFs from integrated network to find the common KEGG pathways and Gene Ontology (GO) biological process terms they regulated. Several previous studies showed that EGFR signaling is one of the potentially targeted pathways for identifying anticancer drugs and treatment strategies for various cancers [61, 62]. The involvement of Wnt signaling pathway in breast cancers have been described previously. Schlange et al had shown that autocrine Wnt signaling controls proliferation and tumor growth through activation of canonical Wnt pathway and EGFR transactivation [63]. Loh et al had shown the important role of this pathway in inhibiting the effects of Tamoxifen in tumor growth [64]. TGF-beta signaling pathway is also

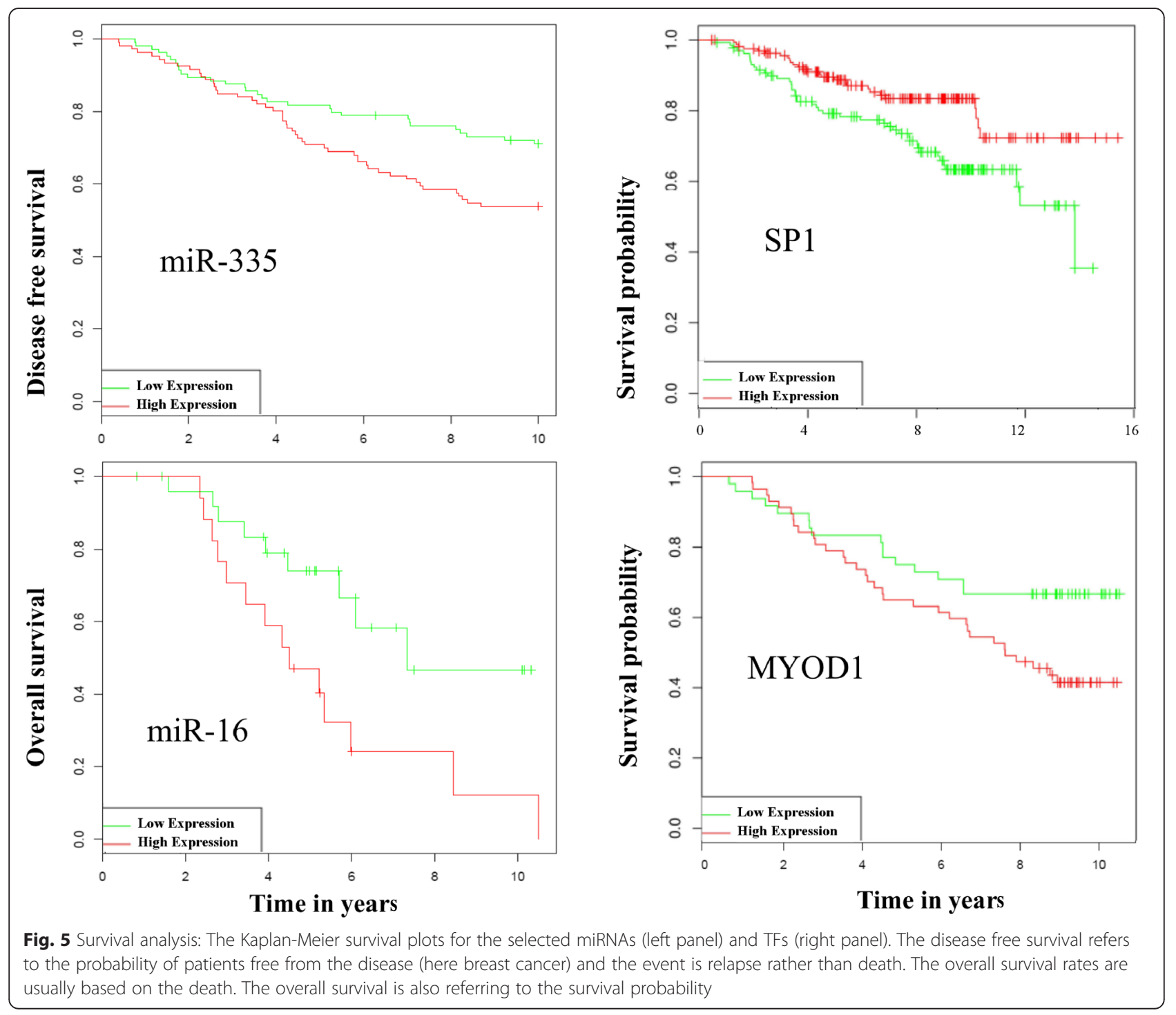


widely studied to identify therapeutic drug targets in many metastatic cancers including breast cancer as this pathway plays a key role in regulating tumor invasion and metastasis [65-67]. Another significantly enriched term was apoptosis. Dysregulation of apoptosis was shown to play key roles in breast cancer [68]. This finding explains the role of EGF as a potential therapeutic target in breast cancers. The MAPK pathway is the central part of the signal transduction initiated by EGF that controls the cellular processes of proliferation and differentiation. This pathway was also highly enriched in the current study and has been widely targeted to find diagnostic and prognostic markers of breast cancer [69]. Although this analysis identified the ErbB as a significantly enriched pathway (as the gene expression data set is obtained from ErbB signaling), to our surprise only 16 out of 1072 differentially expressed TGs were known to be associated with ErbB. This may indicate that our current study identified several new TGs associated with this signaling.

There are few limitations in the current approach used in this study. Firstly, we used only the experimentally verified miRNA-TG regulations from miRTarBase database. This database contains the regulation data retrieved from heterogeneous systems and it may not be accurate for a specific system. Secondly, the NCA approach has very strict criteria on network structure (miRNA-TG, TF-TG) and might have lost few key miRNAs, TFs and their TGs. Thirdly, in spite of the fact that potential prognostic markers for breast cancer in this study were predicted using computational approach only, the validations were based on Kaplan-Meier survival analysis conducted with heterogeneous data sources from clinical trials.

\section{Methods}

\section{Data preprocessing}

The gene expression data used in this study were obtained by measuring the response of MCF7 breast cancer cells treated with epidermal growth factor (EGF) at 17 time points over a time period of $72 \mathrm{~h}$ [70]. The original gene expression data was downloaded from the GEO database with accession number GSE13009. We applied loess normalization within time points and quantile normalization across time points. The expression values were averaged over two replicate measurements. We computed statistical significance, $P$-values based on $\mathrm{t}$ tests by comparing control versus treatment samples at each time point to identify differentially expressed genes (DEGs). The DEGs with a fold change $>1.5$ and $P$-value $<0.05$ at least at two time points were selected for further analysis. To reduce the noise and to smooth the data, we used Fourier transform functions to fit the time-series data [71]. The initial networks were defined using experimentally verified miRNAs, TFs and its interactions with TGs from databases. All the computations were performed using bioinformatics toolbox in MATLAB.

\section{miRNA-TG interactions}

Although several databases are available for predicting miRNA-TG interactions, we chose miRTarBase because it contains manually curated and experimentally verified regulations [72]. We downloaded the regulation data as an adjacency list, which was used in NCA analysis to predict the temporal dynamic activity of miRNAs.

\section{TF-TG interactions}

We collected the experimentally verified TF-TG regulations from TFacts [73], a database containing 6401 experimentally validated regulations between 2720 TGs and 330 TFs. This database includes integrated information from different resources, such as TRED, TRDD, PAZAR NFIregulomeDB and their own experimental predictions. In addition, we retrieved TF-TG interactions from the Chip-X experiments of Transcriptome Browser [74]. This list includes 312 TFs, 13133 TGs and the 173156 interactions among them.

\section{Network component analysis (NCA)}

Network component analysis (NCA) is a computational method for reconstructing hidden regulatory signals (miRNAs activity or TFs activity) from gene expression data with known connectivity information in terms of matrix decomposition [13]. The NCA method can be represented in matrix form as follows:

$$
[E]=[C][T]
$$

where the matrix $[E]$ represents the expression values of genes at various time points, the matrix $[C]$ is the control strength of each miRNA on a target gene (TG), and the matrix $[T]$ represents the activities of all of the miRNAs. The dimensions of $[E],[C]$ and $[T]$ are $\mathrm{N} \mathrm{X} \mathrm{M,}$ $\mathrm{N} X \mathrm{~L}$ and $\mathrm{L} X \mathrm{M}$, respectively. Where, $\mathrm{N}$ is the number of TGs, $M$ is the number of time points or measurement conditions, and $\mathrm{L}$ is the number of miRNAs or TFs.

Based on above formulation, the decomposition of $[E]$ into $[C]$ and $[T]$ can be achieved by minimizing the following objective function:

$$
\begin{aligned}
& \min \|([E]-[C][T])\| \\
& \text { s.t. } C \in \mathrm{Z}_{0}
\end{aligned}
$$

where $Z_{0}$ is the initial connectivity pattern. [C] and [T] are estimated using a two-step least-squares algorithm and are normalized through a nonsingular matrix $[S]$ according to 


$$
[E]=[C][T]=[C][S]\left[S^{-1}\right][T]
$$

To guarantee the uniqueness of the solution for equation (3) up to a scaling factor, certain criteria, termed NCA criteria, must be satisfied:

- The connectivity matrix [C] must have full-column rank

- When a node in the regulatory layer is removed along with all of the output nodes connected to it, the resulting network must be characterized by a connectivity matrix that still has full-column rank

- The $[\mathrm{T}]$ matrix must have full row rank

Using NCA as the reconstruction method, we predicted significant miRNAs, TFs and their temporal activity profiles. The NCA toolbox can be downloaded from here (http://www.seas.ucla.edu/ liaoj/downloads.html).

\section{Integrated approach}

Our integrated approach to study the miRNAs role in the gene regulation networks is composed of several phases (see Fig. 6 and Figs. 1a, 3a). Firstly, we downloaded the gene expression data, pre-processed and combined with connectivity data, run NCA to reconstruct temporal miRNA and TF activities. We clustered the miRNAs that exhibit similar temporal activity patterns and constructed the miRNA-miRNA synergistic network. We then constructed an integrated network by applying the NCA procedure using the differentially expressed genes (DEGs) data that we filtered, and the retrieved TF-TG topology (from the database TFacts and Transcriptome Browser), miR-TG (from the database miRTarBase), and TF-miR (from TransmiR). The interactions in all these databases were experimentally verified interactions. We then extended the resulting network with the predicted synergistic interactions of miRNAs. The detailed description and original sources are provided in the Additional file 7.

\section{The miRNA-miRNA networks}

We computed the pairwise Pearson correlation coefficient between reconstructed activity profiles of all the miRNAs and the number of common TGs between each pair of miRNAs. We assumed a synergistic interaction between a pair of miRNAs if the correlation is greater than 0.7 and common TGs are greater than 3 . The constructed network has 112 miRNAs and 314 synergistic interactions between them. Schematic of the approach is presented in Fig. 3.

The random networks for the comparison purposes are generated in Cytoscape software using 'Randomnetworks' plugin. These networks are created keeping the number of nodes and connections same as the original network.

\section{Pathway and biological processes}

We used DAVID (Database for Annotation, Visualization and Integrated Discovery) with the default settings to find statistically enriched biological pathways. Information related to the pathways was identified from DAVID $[75,76]$. DAVID is a comprehensive set of functional

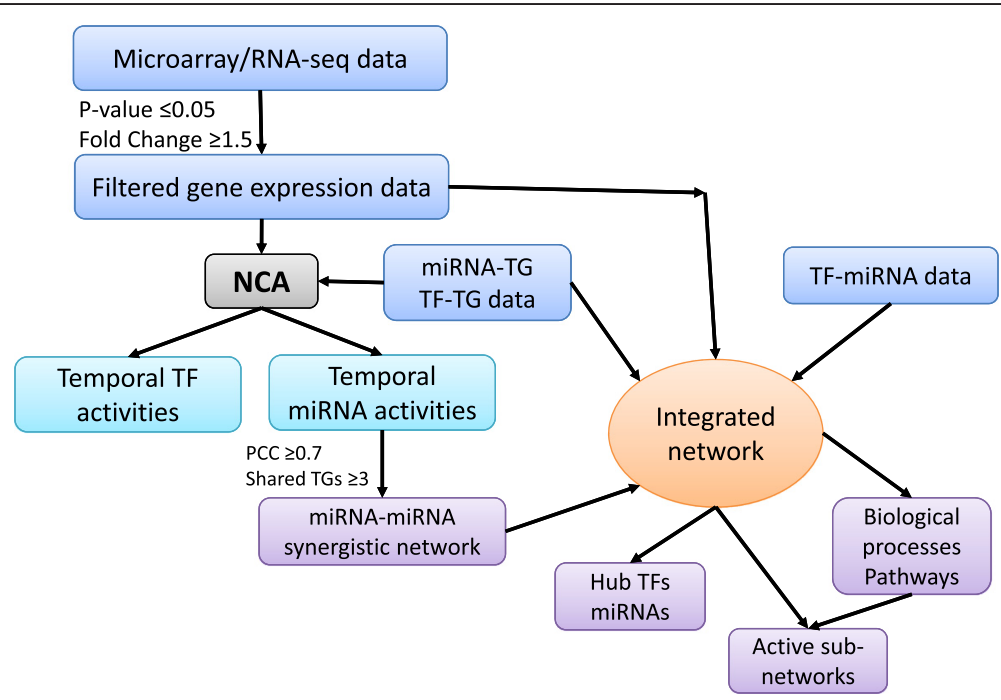

Fig. 6 Schematic of integrated approach: The approach involves the pre-processing of the gene expression data, computing the temporal activities of miRNAs, TFs using NCA and construction of miRNA-miRNA synergistic network, integrated network and identification of significantly enriched biological processes and pathways. PCC stands for Pearson correlation coefficient, TF for transcription factor, TG for target gene and miRNA for micro RNA 
annotation tools for investigators to understand the biological meaning behind a large list of genes. DAVID uses the biological information retrieved from various resources and databases. For instance, information related to pathways is retrieved from KEGG (Kyoto Encyclopedia of Genes and Genomes), PANTHER, BioCarta and REACTOME pathway databases. Pathways and biological processes that had at least 10 DEG members and a $P$-value < 0.001 were considered significant. $P$-values are computed using modified Fisher's exact test based on hyper geometric distribution.

The networks are created using the Cytoscape software tool [77]. All statistical calculations, NCA and clustering were done in Matlab, Mathworks.

\section{Survival analysis}

We conducted survival analysis of miRNAs and TFs using the tools 'MIRUMIR' [78] and 'PPISURV' [79] respectively, both developed by Antonov AV et al. These tools integrate publicly available clinical data such as the GEO repository. Briefly, these tools utilize the rank information of expression profiles of miRNAs and TFs. Patients are divided into low and high expression groups, based on the average expression of the selected miRNAs or TFs. Then, the two distinguished groups of patients along with their survival information are used to identify any significant statistical differences in survival outcome using the statistical packages in R program. The survival outcomes are represented through Kaplan-Meier plots using R. The information about the clinical data source for survival analysis for miRNAs and TFs are provided in Additional file 6B.

\section{Conclusion}

The analytical method we presented here was able to predict the involvement of several key miRNA regulators in processes related to breast cancer. It has also allowed us to explore the role of these regulators in the network and their interactions with TGs and TFs. We demonstrated that this dynamic miRNA-TF network analysis identifies regulation pathways, processes and connections that significantly involved in breast cancer. Furthermore, the identified markers are validated for their potential as prognostic markers for breast cancer though publicly available clinical data and survival analysis. We propose that this analysis can be applied to assist understanding miRNA regulation in other systems as well, suggesting individual miRNAs and entire pathways as target for cancer research.

\section{Additional files}

Additional file 1: The complete list of predicted synergistic interactions of miRNAs is provided as a text file. (ZIP $710 \mathrm{~kb}$ )
Additional file 2: The overlapping synergistic interactions of miRNAs in this study and Sengupta et al study is provided as a tab delimited file (.txt). (DOC $21 \mathrm{~kb}$ )

Additional file 3: The integrated network as a cytoscape file (.cys file) is provided, which can be opened locally by a reader for interactive exploration. (DOC $21 \mathrm{~kb}$ )

Additional file 4: Statistically significant biological pathways affected by differentially expressed TGs in breast cancer cells identified from the PANTHER database is provided in a text file. (DOC $21 \mathrm{~kb}$ )

Additional file 5: Statistically significant biological pathways affected by differentially expressed TGs in breast cancer cells identified from the REACTOME database is provided in a text file. (DOC $21 \mathrm{~kb}$ )

Additional file 6: A: The Kaplan-Meier plots for the miRNAs and TFs. B: The information about the clinical data source for survival analyses for miRNAs and TFs are provided in excel sheet. (DOC $21 \mathrm{~kb}$ )

Additional file 7: The complete details of the integrated procedure with original sources are provided in a pdf file. (DOC $21 \mathrm{~kb}$ )

\section{Competing interests}

The authors declare that they have no competing interests.

\section{Authors' contributions}

NDJ and NB designed the project. NDJ performed all the computations and analyses. NDJ and NB wrote the manuscript. NB supervised the project. Both authors read and approved the final draft.

\section{Acknowledgments}

Authors would like to thank Pavan K Sriram for critical reading of the manuscript. This work was supported by Research Council of Norway through grant number: 70174300 .

Received: 2 June 2015 Accepted: 30 November 2015

Published online: 18 December 2015

\section{References}

1. Chen K, Rajewsky N. The evolution of gene regulation by transcription factors and microRNAs. Nat Rev Genet. 2007;8(2):93-103.

2. He L, Hannon GJ. MicroRNAs: small RNAs with a big role in gene regulation. Nat Rev Genet. 2004;5(7):522-31.

3. Pasquinelli $A E$, Ruvkun $G$. Control of developmental timing by microRNAs and their targets. Annu Rev Cell Dev Biol. 2002;18(1):495-513.

4. Cheng AM, Byrom MW, Shelton J, Ford LP. Antisense inhibition of human miRNAs and indications for an involvement of miRNA in cell growth and apoptosis. Nucleic Acids Res. 2005;33(4):1290-7.

5. Chen C-Z, Li L, Lodish HF, Bartel DP. MicroRNAs Modulate Hematopoietic Lineage Differentiation. Science. 2004;303(5654):83-6.

6. Karp X, Ambros V. Encountering MicroRNAs in Cell Fate Signaling. Science 2005;310(5752):1288-9.

7. Xu P, Guo M, Hay BA. MicroRNAs and the regulation of cell death. Trends Genet. 2004;20(12):617-24.

8. Madden S, Carpenter S, Jeffery I, Björkbacka H, Fitzgerald K, O'Neill L, et al. Detecting microRNA activity from gene expression data. BMC Bioinformatics. 2010;11(1):257.

9. Liang Z, Zhou H, He Z, Zheng H, Wu J. mirAct: a web tool for evaluating microRNA activity based on gene expression data. Nucleic Acids Res. 2011; 39(suppl 2):W139-W144.

10. Mezlini AM, Wang B, Deshwar A, Morris Q, Goldenberg A. Identifying Cancer Specific Functionally Relevant miRNAs from Gene Expression and miRNA-toGene Networks Using Regularized Regression. PLoS One. 2013;8(10):e73168.

11. Cheng C, Li LM. Inferring MicroRNA Activities by Combining Gene Expression with MicroRNA Target Prediction. PLoS One. 2008;3(4):e1989.

12. Schulz MH, Pandit KV, Lino Cardenas CL, Ambalavanan N, Kaminski N, BarJoseph Z. Reconstructing dynamic microRNA-regulated interaction networks. Proc Natl Acad Sci. 2013;110(39):15686-91.

13. Liao JC, Boscolo R, Yang Y-L, Tran LM, Sabatti C, Roychowdhury VP. Network component analysis: Reconstruction of regulatory signals in biological systems. Proc Natl Acad Sci. 2003;100(26):15522-7. 
14. Tran LM, Brynildsen MP, Kao KC, Suen JK, Liao JC. gNCA: a framework for determining transcription factor activity based on transcriptome: identifiability and numerical implementation. Metab Eng. 2005;7(2):128-41.

15. Shao L, Wang L, Wei Z, Xiong Y, Wang Y, Tang K, et al. Dynamic Network of Transcription and Pathway Crosstalk to Reveal Molecular Mechanism of MGd-Treated Human Lung Cancer Cells. PLoS One. 2012;7(5):e31984.

16. Seok J, Xiao W, Moldawer LL, Davis RW, Covert MW. A dynamic network of transcription in LPS-treated human subjects. BMC Syst Biol. 2009;3(78):1-14.

17. Wang J, Qiu X, Li Y, Deng Y, Shi T. A transcriptional dynamic network during Arabidopsis thaliana pollen development. BMC Syst Biol. 2011;5(Supp 3):S8.

18. Fu Y, Jarboe LR, Dickerson JA. Reconstructing genome-wide regulatory network of E. coli using transcriptome data and predicted transcription factor activities. BMC Bioinformatics. 2011;12:233.

19. Hyduke DR, Jarboe LR, Tran LM, Chou KJ, Liao JC. Integrated network analysis identifies nitric oxide response networks and dihydroxyacid dehydratase as a crucial target in Escherichia coli. Proc Natl Acad Sci U S A. 2007:104(20):8484-9.

20. Yang YL, Suen J, Brynildsen MP, Galbraith SJ, Liao JC. Inferring yeast cell cycle regulators and interactions using transcription factor activities. BMC Genomics. 2005;6(90):1-15.

21. Ye C, Galbraith SJ, Liao JC, Eskin E. Using network component analysis to dissect regulatory networks mediated by transcription factors in yeast. PLoS Comput Biol. 2009:5(3):e1000311.

22. Kao KC, Yang YL, Boscolo R, Sabatti C, Roychowdhury V, Liao JC. Transcriptome-based determination of multiple transcription regulator activities in Escherichia coli by using network component analysis. Proc Natl Acad Sci U S A. 2004;101(2):641-6.

23. Rahib L, Sriram G, Harada MK, Liao JC, Dipple KM. Transcriptomic and network component analysis of glycerol kinase in skeletal muscle using a mouse model of glycerol kinase deficiency. Mol Genet Metab. 2009;96(3): $106-12$.

24. Tolle I, Huang X, Akpalu YA, Martin LL. A Modified Network Component Analysis (NCA) Methodology for the Decomposition of X-ray Scattering Signatures. Ind Eng Chem Res. 2009;48(13):6137-44.

25. Brynildsen MP, Liao JC. An integrated network approach identifies the isobutanol response network of Escherichia coli. Mol Syst Biol. 2009; 5(277):277.

26. Buescher JM, Liebermeister W, Jules M, Uhr M, Muntel J, Botella E, et al. Global network reorganization during dynamic adaptations of Bacillus subtilis metabolism. Science. 2012;335(6072):1099-103.

27. Barah P, Jayavelu ND, Mundy J, Bones AM. Genome scale transcriptional response diversity among ten ecotypes of Arabidopsis thaliana during heat stress. Front Plant Sci. 2013;4:532

28. Doni Jayavelu N, Bar N. Dynamics of Regulatory Networks in Gastrin-Treated Adenocarcinoma Cells. PLoS One. 2014;9(1):e78349.

29. Hu X, Guo J, Zheng L, Li C, Zheng TM, Tanyi JL, et al. The Heterochronic microRNA let-7 Inhibits Cell Motility by Regulating the Genes in the Actin Cytoskeleton Pathway in Breast Cancer. Mol Cancer Res. 2013;11(3):240-50.

30. Fonseca-Sanchez MA, Perez-Plasencia C, Fernandez-Retana J, ArechagaOcampo E, Marchat LA, Rodriguez-Cuevas S, et al. microRNA-18b is upregulated in breast cancer and modulates genes involved in cell migration. Oncol Rep. 2013;30(5):2399-410.

31. Iorio MV, Ferracin M, Liu C-G, Veronese A, Spizzo R, Sabbioni S, et al. MicroRNA Gene Expression Deregulation in Human Breast Cancer. Cancer Res. 2005;65(16):7065-70

32. Yu Z, Wang C, Wang M, Li Z, Casimiro MC, Liu M, et al. A cyclin D1/ microRNA 17/20 regulatory feedback loop in control of breast cancer cell proliferation. J Cell Biol. 2008;182(3):509-17.

33. Zeng R-c, Zhang W, Yan X-q, Ye Z-q, Chen E-d, Huang D-p, et al. Downregulation of miRNA-30a in human plasma is a novel marker for breast cancer. Med Oncol. 2013;30(1):1-8.

34. Zhang N, Wang X, Huo Q, Sun M, Cai C, Liu Z, et al. MicroRNA-30a suppresses breast tumor growth and metastasis by targeting metadherin. Oncogene. 2013.

35. Cheng C-W, Wang H-W, Chang C-W, Chu H-W, Chen C-Y, Yu J-C, et al MicroRNA-30a inhibits cell migration and invasion by downregulating vimentin expression and is a potential prognostic marker in breast cancer. Breast Cancer Res Treat. 2012;134(3):1081-93.

36. Gregory PA, Bert AG, Paterson EL, Barry SC, Tsykin A, Farshid G, et al. The miR-200 family and miR-205 regulate epithelial to mesenchymal transition by targeting ZEB1 and SIP1. Nat Cell Biol. 2008;10(5):593-601.
37. Jiang S, Zhang H-W, Lu M-H, He X-H, Li Y, Gu H, et al. MicroRNA-155 Functions as an OncomiR in Breast Cancer by Targeting the Suppressor of Cytokine Signaling 1 Gene. Cancer Res. 2010;70(8):3119-27.

38. Kong W, He L, Coppola M, Guo J, Esposito NN, Coppola D, et al. MicroRNA155 Regulates Cell Survival, Growth, and Chemosensitivity by Targeting FOXO3a in Breast Cancer. J Biol Chem. 2010;285(23):17869-79.

39. Camps C, Buffa FM, Colella S, Moore J, Sotiriou C, Sheldon H, et al. hsa-miR210 Is Induced by Hypoxia and Is an Independent Prognostic Factor in Breast Cancer. Clin Cancer Res. 2008;14(5):1340-8.

40. Jiang $Q$, Wang $Y$, Hao $Y$, Juan $L$, Teng $M$, Zhang $X$, et al. miR2Disease: a manually curated database for microRNA deregulation in human disease. Nucleic Acids Res. 2009;37 suppl 1:D98-D104.

41. Xie B, Ding Q, Han H, Wu D. miRCancer: a microRNA-cancer association database constructed by text mining on literature. Bioinformatics. 2013; 29(5):638-44.

42. Sengupta D, Bandyopadhyay S. Participation of microRNAs in human interactome: extraction of microRNA-microRNA regulations. Mol Biosyst. 2011;7(6):1966-73.

43. Albert R, Barabási A-L. Statistical mechanics of complex networks. Rev Mod Phys. 2002;74(1):47-97.

44. Jeong Y-J, Choi Y, Shin J-M, Cho H-J, Kang J-H, Park K-K, et al. Melittin suppresses EGF-induced cell motility and invasion by inhibiting PI3K/Akt/ mTOR signaling pathway in breast cancer cells. Food and Chemical Toxicology 2014(0)

45. Ginsburg E, Vonderhaar BK. Stimulation of growth of human breast cancer cells (T47D) by platelet derived growth factor. Cancer Lett. 1991;58(1-2): 137-44.

46. Lev DC, Kim SJ, Onn A, Stone V, Nam D-H, Yazici S, et al. Inhibition of Platelet-Derived Growth Factor Receptor Signaling Restricts the Growth of Human Breast Cancer in the Bone of Nude Mice. Clin Cancer Res. 2005; 11(1):306-14.

47. Uluer ET, Aydemir I, Inan S, Ozbilgin K, Vatansever HS. Effects of 5fluorouracil and gemcitabine on a breast cancer cell line (MCF-7) via the JAK/STAT pathway. Acta Histochem. 2012;114(7):641-6.

48. Xu J, Li C-X, Li Y-S, Lv J-Y, Ma Y, Shao T-T, et al. MiRNA-miRNA synergistic network: construction via co-regulating functional modules and disease miRNA topological features. Nucleic Acids Res. 2010.

49. Xu J, Li Y, Li X, Li C, Shao T, Bai J, et al. Dissection of the potential characteristic of miRNA-miRNA functional synergistic regulations. Mol Biosyst. 2013;9(2):217-24.

50. Wu B, Li C, Zhang P, Yao Q, Wu J, Han J, et al. Dissection of miRNA-miRNA Interaction in Esophageal Squamous Cell Carcinoma. PLoS One. 2013;8(9): e73191.

51. Na Y-J, Kim JH. Understanding cooperativity of microRNAs via microRNA association networks. BMC Genomics. 2013;14 Suppl 5:S17.

52. Tavazoie SF, Alarcon C, Oskarsson T, Padua D, Wang Q, Bos PD, et al. Endogenous human microRNAs that suppress breast cancer metastasis. Nature. 2008;451(7175):147-52.

53. Uhlmann S, Mannsperger $H$, Zhang JD, Horvat E-A, Schmidt C, Kublbeck M, et al. Global microRNA level regulation of EGFR-driven cell-cycle protein network in breast cancer. Mol Syst Biol. 2012;8.

54. Li J, Kong X, Zhang J, Luo Q, Li X, Fang L. MiRNA-26b inhibits proliferation by targeting PTGS2 in breast cancer. Cancer Cell Int. 2013;13(1):7.

55. Rivas $M$, Venturutti $L$, Huang $Y-W$, Schillaci R, Huang T, Elizalde $P$. Downregulation of the tumor-suppressor miR-16 via progestin-mediated oncogenic signaling contributes to breast cancer development. Breast Cancer Res. 2012;14(3):R77.

56. Li XF, Yan PJ, Shao ZM. Downregulation of miR-193b contributes to enhance urokinase-type plasminogen activator (UPA) expression and tumor progression and invasion in human breast cancer. Oncogene. 2009;28(44): 3937-48.

57. Yang X, Wang J, Liu S, Yan Q. HSF1 and Sp1 Regulate FUT4 Gene Expression and Cell Proliferation in Breast Cancer Cells. J Cell Biochem. 2014;115(1): $168-78$

58. Zhang Y, Zhao Y, Li L, Shen Y, Cai X, Zhang X, et al. The oncoprotein HBXIP\} upregulates PDGFB\} via activating transcription factor Sp1 to promote the proliferation of breast cancer cells. Biochem Biophys Res Commun. 2013; 434(2):305-10

59. Chen L, Bourguignon LY. Hyaluronan-CD44 interaction promotes C-Jun signaling and miRNA21 expression leading to $\mathrm{BCl}-2$ expression and chemoresistance in breast cancer cells. Mol Cancer. 2014;13(1):52. 
60. Verschoor ML, Verschoor CP, Singh G. Ets-1 global gene expression profile reveals associations with metabolism and oxidative stress in ovarian and breast cancers. Cancer Metab. 2013;1 (1):17.

61. Normanno N, De Luca A, Bianco C, Strizzi L, Mancino M, Maiello MR, et al. Epidermal growth factor receptor (EGFR) signaling in cancer. Gene. 2006; 366(1):2-16.

62. Uberall I, Kolar Z, Trojanec R, Berkovcova J, Hajduch M. The status and role of ErbB receptors in human cancer. Exp Mol Pathol. 2008;84(2):79-89.

63. Schlange T, Matsuda Y, Lienhard S, Huber A, Hynes N. Autocrine WNT signaling contributes to breast cancer cell proliferation via the canonical WNT pathway and EGFR transactivation. Breast Cancer Res. 2007;9(5):R63.

64. Loh YN, Hedditch E, Baker L, Jary E, Ward R, Ford C. The Wnt signalling pathway is upregulated in an in vitro model of acquired tamoxifen resistant breast cancer. BMC Cancer. 2013;13(1):174.

65. Drabsch Y, He S, Zhang L, Snaar-Jagalska B, ten Dijke P. Transforming growth factor-beta signalling controls human breast cancer metastasis in a zebrafish xenograft model. Breast Cancer Res. 2013;15(6):R106.

66. Ganapathy V, Ge R, Grazioli A, Xie W, Banach-Petrosky W, Kang Y, et al. Targeting the Transforming Growth Factor-beta pathway inhibits human basal-like breast cancer metastasis. Mol Cancer. 2010;9(1):122.

67. Zhang L, Zhou F, García de Vinuesa A, de Kruijf Esther M, Mesker Wilma E, Hui L, et al. TRAF4 Promotes TGF- $\beta$ Receptor Signaling and Drives Breast Cancer Metastasis. Mol Cell. 2013;51(5):559-72.

68. Parton M, Dowsett M, Smith I. Studies of apoptosis in breast cancer. BMJ. 2001;322(7301):1528-32.

69. Sparano JA, Moulder S, Kazi A, Vahdat L, Li T, Pellegrino C, et al. Targeted Inhibition of Farnesyltransferase in Locally Advanced Breast Cancer: A Phase I and II Trial of Tipifarnib Plus Dose-Dense Doxorubicin and Cyclophosphamide. J Clin Oncol. 2006;24(19):3013-8.

70. Saeki Y, Endo T, Ide K, Nagashima T, Yumoto N, Toyoda T, et al. Ligandspecific sequential regulation of transcription factors for differentiation of MCF-7 cells. BMC Genomics. 2009;10(545):1-16.

71. Doni Jayavelu N, Bar N. A Noise Removal Algorithm for Time Series Microarray Data. In: Correia L, Reis L, Cascalho J, editors. Progress in Artificial Intelligence, vol. 8154. Berlin: Springer; 2013. p. 152-62.

72. Hsu S-D, Tseng Y-T, Shrestha S, Lin Y-L, Khaleel A, Chou C-H, et al. miRTarBase update 2014: an information resource for experimentally validated miRNA-target interactions. Nucleic Acids Res. 2014;42(D1):D78-85.

73. Essaghir A, Toffalini F, Knoops L, Kallin A, Helden J, Demoulin JB. Transcription factor regulation can be accurately predicted from the presence of target gene signatures in micro array gene expression data. Nucleic Acids Res. 2010;38(11):e120.

74. Lepoivre C, Bergon A, Lopez F, Perumal N, Nguyen C, Imbert J, et al. TranscriptomeBrowser 3.0: introducing a new compendium of molecular interactions and a new visualization tool for the study of gene regulatory networks. BMC Bioinformatics. 2012;13(1):19.

75. Huang DW, Sherman BT, Lempicki RA. Systematic and integrative analysis of large gene lists using DAVID bioinformatics resources. Nat Protoc. 2008;4: 44-57.

76. Dennis G, Sherman B, Hosack D, Yang J, Gao W, Lane HC, et al. DAVID: Database for Annotation, Visualization, and Integrated Discovery. Genome Biol. 2003;4(5):3.

77. Shannon P, Markiel A, Ozier O, Baliga NS, Wang JT, Ramage D, et al. Cytoscape: A Software Environment for Integrated Models of Biomolecular Interaction Networks. Genome Res. 2003;13(11):2498-504.

78. Antonov AV, Knight RA, Melino G, Barlev NA, Tsvetkov PO. MIRUMIR: an online tool to test microRNAs as biomarkers to predict survival in cancer using multiple clinical data sets. Cell Death Differ. 2013;20(2):367.

79. Antonov AV, Krestyaninova M, Knight RA, Rodchenkov I, Melino G, Barlev NA. PPISURV: a novel bioinformatics tool for uncovering the hidden role of specific genes in cancer survival outcome. Oncogene. 2014;33(13):1621-8.

\section{Submit your next manuscript to BioMed Central and we will help you at every step:}

- We accept pre-submission inquiries

- Our selector tool helps you to find the most relevant journal

- We provide round the clock customer support

- Convenient online submission

- Thorough peer review

- Inclusion in PubMed and all major indexing services

- Maximum visibility for your research

Submit your manuscript at www.biomedcentral.com/submit
() BioMed Central 